\title{
Energy transfer between a biological labelling dye and gold nanorods
}

\author{
Chris Racknor ${ }^{1}$, Mahi R. Singh ${ }^{1, a)}$, Yinan Zhang ${ }^{2}$, David J.S. Birch ${ }^{2}$, and Yu Chen $^{2, b)}$. \\ ${ }^{1}$ Department of Physics and Astronomy, Western University, \\ London, Ontario, Canada N6A $3 K \%$. \\ ${ }^{2}$ Photophysics Group, Centre for Molecular Nanometrology, \\ Department of Physics, SUPA, \\ University of Strathclyde, John Anderson Building, \\ 107 Rottenrow, Glasgow G4 ONG, UK \\ ${ }^{a)}$ msingh@uwo.ca, ${ }^{b)}$ y.chen@strath.ac.uk
}

\begin{abstract}
We have demonstrated energy transfer between a biological labeling dye (Alexa Fluor 405) and gold nanorods experimentally and theoretically. The fluorescence lifetime imaging microscopy and density matrix method are used to study a hybrid system of dye and nanorods under one- and two-photon excitations. Energy transfer between dye and nanorods via the dipole-dipole interaction is found to cause a decrease in the fluorescence lifetime change. Enhanced energy transfer from dye to nanorods is measured in the presence of an increased density of nanorods. This study has potential applications in fluorescence lifetime-based intra-cellular sensing of bio-analytes as well as nuclear targeting cancer therapy.
\end{abstract}

PACS: 73.21.La, 71.35.Cc

PACS numbers: 73.21.La,71.35.Cc

\section{INTRODUCTION}

Interest in the development of nanoscale optoelectronic devices has progressed recently by combining different nanomaterials into hybrid structures (Achermann et al 2010, Artuso et al 2010, Sadeghi et al 2009, Cheng et al 2007, Durach et al 2008, Luther et al 2011). These hybrid systems are made from the combination of two or more nano-scale components such as quantum dots, graphene, carbon nanotubes, semiconductor nanowires, metallic nanoparticles, metallic nanowires and nanobiomaterials (DNA) to enhance or decrease optical interactions and effects. These hybrid nanostructures could prove to be potentially strong tools for the future of biotechnology (Mulder et al 2009, Gao et al 2009, Costi et al 2010) and solar energy collection (Huynh et al 2002, Park et al 2011).

To fuel the current revolution in life science imaging new experimental tools that enable the manipulation of biomolecules and the study of biological processes at the molecular level are needed. Biomolecule-nanoparticle hybrid systems represent functional information gathering units for biological applications. To create fluorescent probes there are many types of potential hybrid matches such as organic dye-metal (Kumar 2005) and nanoparticle-enzyme (Willner et al 2007). Specifically magnetic hybrid nanostructures have applications as contrast agents in MRI technology (Quarta et al 2007). Fluorescent dyes are combined with quantum dots in hybrid systems as photosensitizers for photodynamic therapy in cancer studies. A Methylene blue (dye)-semiconductor nanocrystal match points toward an improvement in the cancer cell kill efficiency (Rakovich et al 2010). A system of two linear linked dye molecules to metal oxide nanoparticles has been made to be a dye-sensitized so- lar cell covering a wavelength range from 400 to $950 \mathrm{~nm}$ (Park et al 2011). Organic dye molecules and noble metal nanoparticles show improved fluorescence quenching efficiency and photostability as resonant energy transfer systems (Dulkeith et al 2002).

Also, Silver colloids selectively bound with thiolated 23-mer oligonucleotide in a hybrid system with fluorescein were studied. Their interaction made for an observed increase in fluorescence emission (Lukomska et al 2004). An organic dye(SAMSA)-gold nanoparticle hybrid system has been recently found to have random photoactivated fluorescence blinking whose rate depends on the size of the nanoparticles. These properties have the potential for use as light emitting nanostructures (Cannone et al 2007).

The aim of this paper will be on a hybrid system consisting of Alexa Fluor 405 dye (AFD), a biological fluorescent labeling dye, and gold nanorods (GNRs). We will be concerning ourselves with the energy transfer rate arising from the interaction of optical exctiations between these components of the system. These interactions are strong when the AFD and GNR are in close proximity and their optical excitation frequencies are resonant with each other.

The optical excitations in the metallic nanorods are the collective oscillations of conduction band electrons, called surface plasmon polaritons. Polaritons arise from the dielectric contrast between the metal and an embedded dielectric media while the resonant frequencies of SPPs are dictated by the shape and size of the metallic system. The future of nanophotonic devices and circuits utilizing the polaritons is expected to be significant in optical signals processing, nanoscale optical devices, and near-field microscopy with nanoscale resolution (Gramotnev et al 2010). 


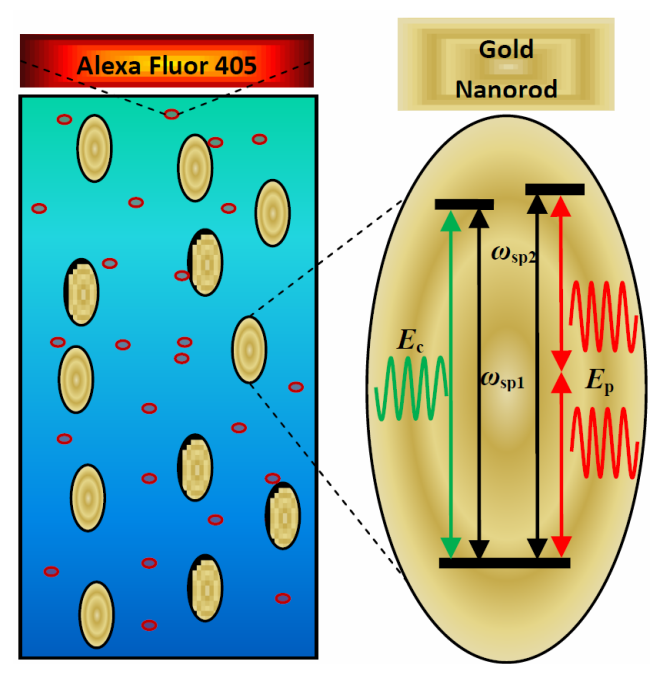

FIG. 1: Schematic diagram of Alexa Fluor405-gold nanorod hybrid system.

Two-photon effects are well known phenomena and have a wide range of applications (Hayat et al 2011), specifically to bioscience imaging (Helmchen et al 2005). Two-photon excitation has advantage over one-photon excitation because it has higher spatial resolution, deeper penetration and less photo-damage. Nanoparticles made of pure noble metals have high electron polarizability and produce enhanced local electric fields that are particularly important for nonlinear optical processes. Multiphoton photoluminescence has been studied in nanostructured noble metals (Schuck et al 2005, Bouhelier et al 2005, Imura et al 2006) and it is found that it is more sensitive to the local field than single-photon luminescence. One- and two-photon induced fluorescence of Pacific Blue (PB)-labeled human serum albumin (HSA) has been studied in the presence of different size silver colloids. The two-photon excitation showed stronger increases in brightness compared to one-photon excitation (Lukomska et al 2005).

AFDs can act as a cell and tissue labels in fluorescence microscopy and cell biology. The AFD is efficiently activated by lasers in the wavelength range near $405 \mathrm{~nm}$ (Bates et al 2007). This work investigates the energy transfer rates for situations where the AFD is excited by one- and two-photon absorption, respectively. Alexa Fluor dyes are generally more stable, brighter, and less pH-sensitive than common dyes (e.g. fluorescein, rhodamine) of comparable excitation and emission (Panchuk-Voloshina et al 1999).

The GNRs are synthesized by the seeded growth method with a typical length about $50 \mathrm{~nm}$ and aspect ratio about 3.5 obtained from SEM measurement. For energy transfer studies, we prepared a GNR and AFD hybrid system. A schematic of the hybrid solution is presented in fig. 1. The first sample S0-1 was prepared by combining the mixture solution of $500 \mu \mathrm{l} 0.21 \mathrm{nM}$ GNRs

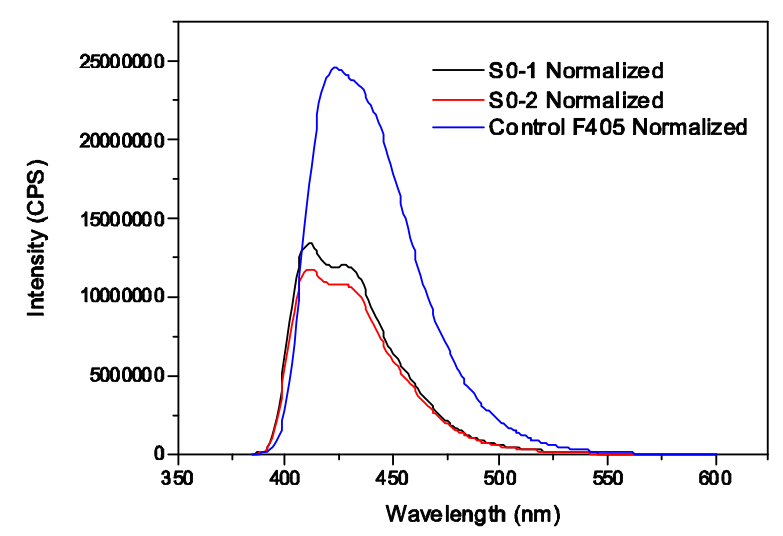

FIG. 2: The spectrum of Alexa Fluor 405 and mixture samples, S0-1 and S0-2, excited at 385nm.

and $150 \mu \mathrm{l} 2 \mu \mathrm{M}$ AFD making for an AFD/GNR ratio of 3000:1. Similarly, a second sample S0-2 was prepared by mixing a solution of $500 \mu \mathrm{l} 0.21 \mathrm{nM}$ GNRs and $300 \mu \mathrm{l} 2 \mu \mathrm{M}$ AFD for a ratio of 6000:1. Samples were placed in an imaging chamber for microscopy studies. Fluorescence-lifetime imaging microscopy (FLIM) experiments were performed using a confocal microscope (LSM 510, Carl Zeiss) equipped with a time-correlated single photon counting module (SPC-830, Becker \& Hickl $\mathrm{GmbH}$ ). A femtosecond Ti:Sapphire laser (Chameleon, Coherent) with a laser pulse repetition rate of $80 \mathrm{MHz}$ and duration less than 200 fs. Emission was collected using a 60 water immersion objective (N.A. $\left.\frac{1}{4} 1.0\right)$ and a bandpass filter with a transmission window from $390 \mathrm{~nm}$ to $465 \mathrm{~nm}$. The GNRs have a longitudinal plasmon mode centred at around $850 \mathrm{~nm}$ and a transverse band at 525 nm.

We have examined the interaction between GNRs and AFD in a mixed solution by monitoring the fluorescence lifetime change. Here sample F405 is the pure AFD and samples S0-1 and S0-2 are AFD-GNR hybrids. Fig.2 displays the comparison of AFD fluorescence intensity in S0-1, S0-2 and reference control solution excited at $385 \mathrm{~nm}$. All measurements have been normalized in term of dye concentration so that direct comparison can be made. Both S0-1 and S0-2 showed significant drop in fluorescence intensity. Furthermore, S0-2 had a further decrease in intensity compared to S0-1.

In two separate experiments the one-photon and twophoton fluorescence lifetime change in samples F405, S01 , and S0-2 were observed under excitation from two different lasers. In the one-photon experiment the laser with wavelength $374 \mathrm{~nm}$ is used and emissions were collected with longpass $405 \mathrm{~nm}$ filter. One-photon excitation fluorescence decay curves of all samples are presented in fig. 3. Under one-photon excitation $(374 \mathrm{~nm})$ the GNRs do not emit light, so the decay curve of mixture solution 
can be directly compared to that of the pure F405 sample. All AFD-GNR hybrid samples (S0-2 and S0-1) show significant decrease in decay time. This lifetime reduction alongside a fluorescence intensity decrease suggests an energy transfer between AFD and GNRs.

In the two-photon excitation experiments a laser with wavelength 810nm (Ti: Saphire) is used and both AFD and GNRs emit luminescence. The GNRs emit luminescence with a lifetime shorter than the system response time (Zhang et al 2010). In fig. 4 we show normalized decay curves (after subtracting the effect from nanorods) of F405, S0-1, and S0-2 under two-photon excitation. Emission from GNR solution was used as system response (or prompt) to achieve good-quality fitting results. More significant decrease in lifetime (down to $2.6 \mathrm{~ns}$ from $3.7 \mathrm{~ns}$ ) has been found, which suggests an enhanced energy transfer process when excitation wavelength matches with longitudinal surface plasmon resonance of GNRs. This enhanced energy transfer under two-photon excitation shows a benefit to using gold nanorods in energy transfer pairs in comparison with gold nanospheres.

In this paper we have considered the dipole-plasmon interaction between the dye and metal nanoparticles for the two-photon process, we call it a dipole-dipole interaction. In the literature the surface energy transfer (SET) theory has been widely used to explain experimental data (Zhang et al 2011, Sen et al 2007, Sen et al 2010, Yun et al 2005). The SET theory gives a $1 / d^{4}$ distance dependence behaviour where $d$ would be the distance between the dye and a metallic nanoparticle. This theory has been developed for a one-photon process and it is valid for a special case when the one-dimensional (1D) localized plasmons are interacting with a dipole. The 1D localized plasmons are present in two-dimensional metallic surface with a semi-infinite positive back-ground (Persson et al 1982). In the present work, the plasmons in the nanorods are localized in all three dimensions because the size of the nanorod in all three dimensions is smaller than the wavelength of the incident light. In nanorods there is no semi-infinite positive back-ground. Hence the SET theory cannot be applied to the present case and we have developed a theory for the two-photon energy transfer between a dye and a nanorod as follows.

In the above experiments one-photon and two-photon processes are studied in the AFD. The AFD acts as a donor nanoparticle and the GNR acts as an acceptor nanoparticle in the hybrid system. According to selection rules, the one-photon excitation occurs only when the ground state and the excited state have the opposite parity. Similarly the two-photon excitation occurs only when the ground and excited states have the same parity. Therefore we consider that the AFD must have one ground state and two excited states with opposite parity. We model that the AFD's three states which are denoted as $|1\rangle,|2\rangle$, and $|3\rangle$ where $|1\rangle$ is the ground state and $|2\rangle$ and $|3\rangle$ are excited states. The $374 \mathrm{~nm}$ laser is represented by $E_{c} \cos \left(\omega_{c} t\right)$ which excites the one-photon transition from $|1\rangle$ to $|3\rangle$ (i.e. $\omega_{c} \simeq \omega_{13}$ ). The $810 \mathrm{~nm}$ laser is $E_{p} \cos \left(\omega_{p} t\right)$ and it excites a two-photon transition from from $|1\rangle$ to $|2\rangle$ (i.e. $2 \omega_{p} \simeq \omega_{12}$ ). We also consider that the $374 \mathrm{~nm}$ and $810 \mathrm{~nm}$ lasers excite one- and twosurface plasmon polaritons in the GNR with frequency $\omega_{s p 1}=\omega_{c}$ and $\omega_{s p 2}=2 \omega_{p}$, respectively.

\section{THEORY}

One- and two-photon excitation dipoles are induced in the AFD and GNR. They interact with each other via the dipole-dipole interaction (DDI). Due to the DDI, energy transfer occurs from AFD to GNR and can be calculated as (Meystre et al 2007)

$$
W_{d \rightarrow g}^{i}=\frac{1}{V_{g}}\left\langle\int\left[j_{g}^{i} \cdot E_{d}^{i}\right] d V\right\rangle .
$$

where $i=1 p h$ and $2 p h$. Here $j_{g}^{i}$ is the induced current and $V_{g}$ is the volume of GNR. The arrowed brackets, \langle\rangle , signify a time average over the period of the oscillating laser fields. The fields $E_{d}^{1 p h}$ and $E_{d}^{2 p h}$ are the dipole elctric fields created by the AFD due to the one- and two-photon excitations at the location of the GNR, respectively. They are written as

$$
E_{d}^{1 p h}=\frac{g P_{d}^{1 p h}}{\left(4 \pi \epsilon_{0} \epsilon_{b}\right) \epsilon_{B} R^{3}}, E_{d}^{2 p h}=\frac{g P_{d}^{2 p h}}{\left(4 \pi \epsilon_{0} \epsilon_{b}\right) \epsilon_{B} R^{3}},
$$

where $P_{d}^{1 p h}$ and $P_{d}^{2 p h}$ are induced dipoles in the AFD due to the one- and two-photon excitations. Here $\epsilon_{B}=$ $\left(2 \epsilon_{b}+\epsilon_{d}\right) / 3 \epsilon_{b}$ where $\epsilon_{b}$ and $\epsilon_{d}$ are the dielectric constants of the host substance, i.e. water, and the AFD. Here $g$ is called the polarization parameter with $g=2$ or $=$ -1 . The center-to-center distance between the AFD and GNR is denoted as $R$.

The one- and two-photon polarization in AFD can be calculated by using the density matrix method as (Meystre et al 2007)

$$
P_{d}^{1 p h}=\mu_{13} \varrho_{13}, P_{d}^{2 p h}=k_{12} \varrho_{12} E_{p},
$$

The physical parameters $\varrho_{12}$ and $\varrho_{13}$ are the density matrix elements between states $|1\rangle$ and $|2\rangle$ and $|1\rangle$ and $|3\rangle$, respectively. Here $\mu_{13}$ and $k_{12}$ are the one-photon and two-photon dipole moments and are given in reference (Sarid et al 2010).

The current density $j_{g}^{i}$ in the GNR is calculate by differentiating the polarization $P_{g}^{i}$ with time as such, $j_{g}^{i}=\left(d P_{g}^{i} / d t\right) / V_{g}$.

The nonlinear two-photon polarization $P_{g}^{1 p h}$ in the GNR can be calculated by using the quasi-static approximation (Nanotny et al 2006). We consider a GNR of an ellipsoidal shape with major axis $l$ and minor axis $r$. The electric field seen by the GNR are $E_{c}$ and $E_{d}^{1 p h}$, for onephoton process and $E_{p}$ and $E_{d}^{2 p h}$ for two-photon process. 
The resultant polarization of the GNR is

$$
P_{g}^{1 p h}=\chi_{1}\left(E_{c}+E_{d}^{1 p h}\right), P_{g}^{2 p h}=\chi_{1} E_{d}^{2 p h}+\chi_{2} E_{p}^{2},
$$

where

$\chi_{1}=\frac{4 \pi \epsilon_{0} \epsilon_{b} V_{a}\left[\epsilon_{m}-\epsilon_{b}\right]}{3 \epsilon_{b}+3 \varsigma_{l, r}(u)\left(\epsilon_{m}-\epsilon_{b}\right)}, \chi_{2}=\frac{m_{e} \zeta \chi_{g 1}^{2}\left(\omega_{p}\right) \chi_{g 1}\left(2 \omega_{p}\right)}{e^{3}}$,

and where $\epsilon_{m}(\omega)$ is dielectric constant of the GNR and taken from Ref. (Meystre et al 2007). Here $m_{e}, \zeta$, and $e$ are the mass of an electron, coupling constant with unit $\left(m^{-1} s^{-2}\right)$ and the charge of the electron, respectively. Parameter $\varsigma_{l, r}(u)$ is the depolarization factor and is defined in Ref. (Cox et al 2010) and it depends on the shape of the nanorod $(u=r / l)$.

With the help of eqns (2-4), eqn (1) the energy transfer rate calculated as

$$
\begin{aligned}
W_{d \rightarrow g}^{1 p h} & =\frac{\omega_{13}}{2}\left[\operatorname{Im}\left(\chi_{1}^{*}\right)\left|E_{d}^{1 p h}\right|^{2}+\operatorname{Im}\left(\chi_{1}^{*} E_{c}^{*} E_{d}^{1 p h}\right)\right](6 \mathrm{a}) \\
W_{d \rightarrow g}^{2 p h} & =\frac{\omega_{12}}{2}\left[\operatorname{Im}\left(\chi_{1}^{*}\right)\left|E_{d}^{2 p h}\right|^{2}+\operatorname{Im}\left(\chi_{2}^{*} E_{d}^{2 p h}\right) \mid E_{p}(\hat{b}]\right)
\end{aligned}
$$

where $W_{d \rightarrow g}^{1 p h}$ and $W_{d \rightarrow g}^{2 p h}$ are the energy transfer rate due to the one- and two-photon DDI from the AFD to the GNR. Note that energy rate depends on the one- and two-photon DDI between the AFD and GNR. A similar expression to eqn. (6a) for the one-photon process has been calculated in Ref. (Artuso et al 2010). This expression depends on the density matrix element $\rho_{13}$ which are also been calculated in that work. The two-photon energy transfer rate depends on density matrix element $\rho_{12}$ which is calculated as follows.

We have calculated that polarization induced in the GNR in eqn. (4). This polarization creates a dipole field near the AFD and is written as

$$
E_{g}^{2 p h}=\frac{g P_{g}^{2 p h}}{\left(4 \pi \epsilon_{0}\right) \epsilon_{B} R^{3}} .
$$

The AFD and GNR interact via DDI. Using the above expression the DDI Hamiltonian of the hybrid system is

$$
H_{d d i}=\hbar\left(\Lambda_{2 p}+\Lambda_{d d i}\right) \sigma_{21}+h c,
$$

where

$$
\Lambda_{2 p}=\frac{g k_{12} \varrho_{12} \chi_{2} E_{p}^{3}}{\left(4 \pi \epsilon_{0} \epsilon_{b}\right) \epsilon_{B} R^{3}}, \Lambda_{d d i}=\frac{g^{2} \chi_{1}\left(k_{12} \varrho_{12} E_{p}\right)^{2}}{\left(4 \pi \epsilon_{0} \epsilon_{b}\right)^{2} \epsilon_{B}^{2} R^{6}} .
$$

Here $h c$ stands for the Hermitian conjugate and $\sigma_{i j}=$ $|i\rangle\langle j|$ is called the creation operator from state $|i\rangle$ to $|j\rangle$. Note that DDI Hamiltonian depends on $\chi_{1}$ and $\chi_{2}$ whose denominator becomes zero at $\omega_{s p}=\omega_{12}$. This means that the DDI is strongest when the frequencies of AFD and GNR are resonant $\left(\omega_{s p 2}=\omega_{12}\right)$.
We use the density matrix method developed for the and one- and two-photon process in Ref. (Meystre et al 2007). Using eqn. (8) and two-photon rotating wave approximation we obtained the following expressions for density matrix elements

$$
\begin{gathered}
\dot{\rho_{22}}=-\gamma_{2} \rho_{22}+i \Omega_{12} \rho_{12}-i \Omega_{12}^{*} \rho_{21} \\
\stackrel{\bullet}{\rho_{12}}=-\left[\gamma_{2} / 2-i\left(\delta_{21}+\delta_{S}\right)\right] \rho_{12}+i \Omega_{12}^{*}\left(\rho_{22}-\rho_{11}\right)
\end{gathered}
$$

where $\Omega_{12}=\left(\Omega_{2 p}+\Lambda_{2 p}+\Lambda_{d d i}\right), \rho_{11}+\rho_{22}=1, \rho_{12}=$ $\rho_{21}^{*}$, and $\delta_{S}=\beta_{S}\left|\Omega_{12}\right|$. The detuning parameter here is $\delta_{21}=2 \omega_{p}-\omega_{12} \cdot \gamma_{2}$ is the spontaneous decay rate for states $|2\rangle$ and $\beta_{S}=\left(k_{b b}-k_{a a}\right) / 4 k_{a b}$ where $k_{a a}, k_{b b}, k_{a b}$ are the two-photon coefficients (Meystre et al 2007).

The energy transfer from AFD to GNR is measured via the fluorescence of the AFD. The energy transfer is observed as a decrease of the AFD's fluorescence intensity and increase of the GNR's. In the fluorescence measurement, the intensity emission from the AFD is measured in time. The decay time of AFD in the presence of GNRs is given as

$$
\begin{aligned}
& I_{d \rightarrow g}^{1 p h}=I_{0} \exp \left(-\gamma_{3} t-\gamma_{1 p h} t\right) ; \\
& I_{d \rightarrow g}^{2 p h}=I_{0} \exp \left(-\gamma_{2} t-\gamma_{2 p h} t\right)
\end{aligned}
$$

where $\gamma_{3}$ and $\gamma_{2}$ are radiative decay rates of the AFD for one- and two-photon in the absence of the GNRs, respectively. Similarily $\gamma_{1 p h}$ and $\gamma_{2 p h}$ are the nonradiative decay rates due to one- and two-photon energy transfer from the AFD to the GNR. It has found that the radiative and nonradiative decay rates are related to the energy transfer rate of the AFD in the absence and in the presence of the GNR, respectively by the following identity (Nanotny et al 2006)

$$
\frac{\gamma_{1 p h}}{\gamma_{3}}=\frac{W_{d \rightarrow g}^{1 p h}}{W_{\text {rad }}^{1 p h}}, \frac{\gamma_{2 p h}}{\gamma_{2}}=\frac{W_{d \rightarrow g}^{2 p h}}{W_{\text {rad }}^{2 p h}},
$$

where $W_{r a d}^{1 p h}$ and $W_{\text {rad }}^{2 p h}$ are one- and two-photon energy transfer rate of the AFD in the absence of the GNR, they are calculated as (Nanotny et al 2006)

$$
W_{\text {rad }}^{1 p h}=\hbar \omega_{13} \rho_{33}^{0} \gamma_{3}, W_{\text {rad }}^{2 p h}=\hbar \omega_{12} \rho_{22}^{0} \gamma_{2},
$$

where $\rho_{22}^{0}$ and $\rho_{33}^{0}$ are the density matrix elements for $|2\rangle$ and $|3\rangle$ in the absence of the GNR and measure the population of $|2\rangle$ and $|3\rangle$, respectively. They are evaluated from eqn. (9) by neglecting the DDI Hamiltonian. By putting eqns 11 and 12 into $10 \mathrm{a} \& 10 \mathrm{~b}$ we get the oneand two-photon florescence intensities.

The AFD-GNR pair does not exist alone but in an ensemble of AFD-GNR pairs. We consider that the AFDs and GNRs are separated according to the Gaussian distribution as

$$
P(R)=\frac{1}{\sqrt{2 \pi} \sigma} \exp \left[-\frac{1}{2}\left(\frac{R_{a v}-R}{\sigma}\right)^{2}\right],
$$




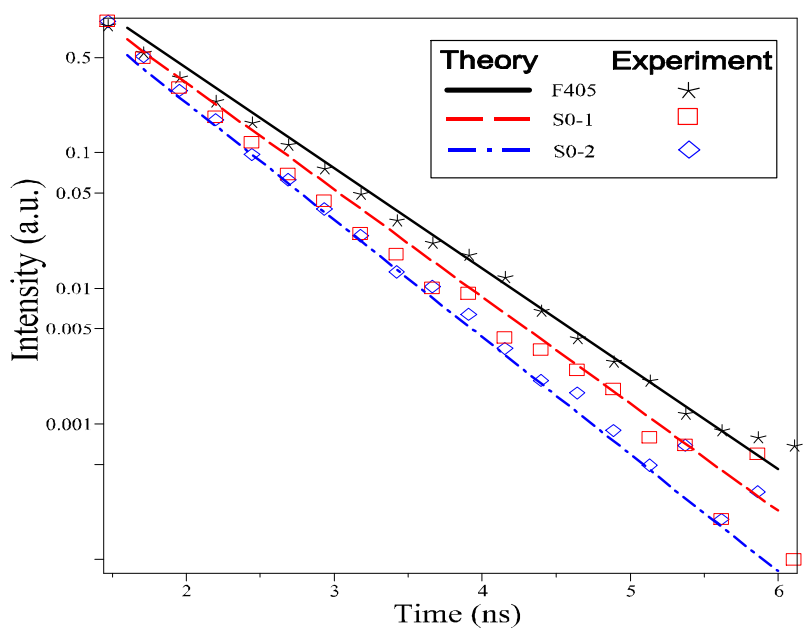

FIG. 3: Normalized theoretical and experimental fluorescence intensity under one-photon excitation.

where $R_{a v}$ is the average distance between a AFD and GNR pair and $\sigma$ is the standard deviation. The florescence intensity decay is now the summation of all accessible distances between the AFD and GNR such that

$$
I_{d \rightarrow a}=\int_{0}^{\infty} d^{3} R P(R) I_{d \rightarrow a}(R) .
$$

This is simply the weighted average of the decay for each AFD-GNR distance.

\section{RESULTS AND DISCUSSION}

For simulations sake the $374 \mathrm{~nm}$ and $810 \mathrm{~nm}$ laser fields are taken to be resonant with their transitions, therefore $\delta_{12}=\delta_{13}=0$. This is fair given that the intensities under comparison are normalized. The three sample solutions contain the hybrid system in an aqueous solution. For this reason, the background dielectric constant is that of water, $\epsilon_{b}=1.77$. For the F405 three level model the dipole moment $\mu_{13}=0.004 \mathrm{e} \mathrm{nm}$ and two-photon coupling constant $k_{12}=2 \cdot 10^{-36} \mathrm{mC}^{2} / \mathrm{N}, g=-1$. The radius of the nanorods is taken to be $r=50 \mathrm{~nm}$ and $u=1 / 3$ while the radius for F405 molecule is $1 \mathrm{~nm}$.

Using eqn. (10a) we have calculated the one-photon fluorescence intensity for the F405-GNR hybrid system . The results are plotted in fig. 3 by solid, dashed, and dashed-dotted lines for three samples F405, S0-1 and $\mathrm{S} 0-2$, respectively. A good agreement between theory and experiment is found. The fluorescence intensity for the F405 sample is calculated by neglecting DDI terms, $W_{d \rightarrow g}^{1 p h}=0$, in eqn. (10a). This gives us the decay rate for the AFD in the absence of the GNR $\gamma_{3}^{-1}=0.588$ ns. For samples S0-1 and S0-2 the nonradiative decay rates were found as $\gamma_{1 p h}^{-1}=0.04 \mathrm{~ns}$ and $\gamma_{1 p h}^{-1}=0.10 \mathrm{~ns}$, respectively. Both mixture solution samples show significant decrease

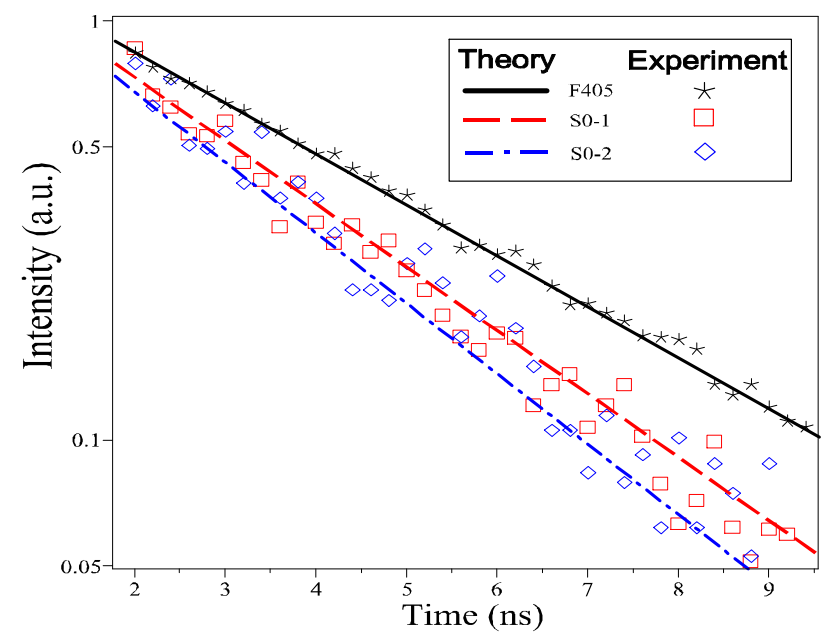

FIG. 4: Normalized theoretical and experimental fluorescense intensity under two-photon excitation.

in their decay rates. The decrease in the decay rate is due to energy transfer from the AFD to the GNR due to the one-photon DDI. Note that the decrease in the decay time in S0-2 is larger than that of the S0-1. This means the DDI interaction in the S0-2 sample is larger than in the S0-1 sample. The average centre-to-centre distance between the AFD and GNR for S0-1 and S0-2 are estimated at $R_{a v}=71 \mathrm{~nm}$ and $R_{a v}=61 \mathrm{~nm}$, respectively. This also shows that the DDI in S0-2 is larger than that of the S0-1 since the DDI is inversely proportional to the distance between the AFD and GNR.

The numerical simulations for two-photon fluorescence intensity is performed by using eqn. (10b) and results are shown in fig. 4. The solid, dashed and dasheddotted lines again represent Fluor405, S0-1 and S0-2. Strong agreement between theory and experiments is again found. The F405 is calculated for $W_{d \rightarrow g}^{2 p h}=0$ and the radiative two-photon decay rate is calculated $\gamma_{2}^{-1}=3.57$ ns. The nonradiative two-photon decay rates for S0-1 and S0-2 are $\gamma_{2 p h}^{-1}=0.87 \mathrm{~ns}$ and $\gamma_{2 p h}^{-1}=1.37 \mathrm{~ns}$, respectively.

\begin{tabular}{|c|c|c|}
\hline & $\gamma_{2}^{-1}$ & $\gamma_{3}^{-1}$ \\
\hline F405 & $3.57 \mathrm{~ns}$ & $0.588 \mathrm{~ns}$ \\
\hline \multicolumn{3}{|c|}{ non-radiative decay rat } \\
\hline & $\gamma_{1 p h}^{-1}$ & $\gamma_{2 p h}^{-1}$ \\
\hline S0-1 & $0.04 \mathrm{~ns}$ & $0.87 \mathrm{~ns}$ \\
\hline S0-2 & $0.10 \mathrm{~ns}$ & $1.37 \mathrm{~ns}$ \\
\hline
\end{tabular}

Decrease in the decay rates for both samples is consistent with the one-photon case and is motivated by the two-photon energy transfer from the AFD to the GNR. The decrease in the decay time in $\mathrm{S} 0-2$ is again larger than that of the S0-1 and therefore its DDI interaction is larger. These results are consistent with the result from a FLIM study in cell culture (Zhang et al 2011). 


\section{CONCLUSION}

The energy transfer between biological labeling dye (Alexa Fluor 405) and gold nanorods has been studied experimentally and theoretically. Fluorescence lifetime imaging microscopy and the density matrix method are applied to study a hybrid system of dye and nanorods under one- and two-photon excitations. The DDI interaction between AFD and GNR causes a decrease in the fluorescence lifetime change. The energy transfer provides detailed information in biological studies using metallic nanoparticles as fluorescence probes, especially when combined with the advantages of two-photon excitation microscopy (Zhang et al 2011). The present study has potential applications in FRET imaging and fluorescence lifetime-based intra-cellular sensing of bio-analytes such as metal ions, proteins, as well as nuclear targeting cancer therapy.
[1] Achermann M 2010 Exciton-Plasmon Interactions in Metal-Semiconductor Nanostructures J. Phys. Chem. Lett. 1, 2837

[2] Artuso R D and Bryant G W 2010 Strongly coupled quantum dot-metal nanoparticle systems: Excitoninduced transparency, discontinuous response, and suppression as driven quantum oscillator effects Phys. Rev. B 82, 195419

[3] Sadeghi S M, Deng L, Li X and Huang W-P Plasmonic (thermal) electromagnetically induced transparency in metallic nanoparticle--quantum dot hybrid systems Nanotechnology 20, 365401

[4] Cheng M-T, Liu S-D, Zhou H-J, Hao Z-H, and Wang Q-Q 2007 Coherent exciton-plasmon interaction in the hybrid semiconductor quantum dot and metal nanoparticle complex Opt. Lett. 32, 2125

[5] Durach M, Rusina A, Klimov V I, and Stockman M I 2008 Nanoplasmonic Renormalization and Enhancement of Coulomb Interactions New J. Phys. 10, 105011

[6] Luther J M, Jain P K, Ewers T, and Alivisatos A P 2011 Localized surface plasmon resonances arising from free carriers in doped quantum dots Nat. Mater. 10, 361

[7] Mulder W J M, Strijkers G J, van Tilborg G A F, Cormode D P, Fayad Z A, and Nicolay K 2009 Nanoparticulate assemblies of amphiphiles and diagnostically active materials for multimodality imaging. Acc. Chem. Res. 42(7), 904-914

[8] Gao J, Gu H, and Xu B 2009 Multifunctional Magnetic Nanoparticles: Design, Synthesis, and Biomedical Application Acc. Chem. Research 42, 1097

[9] Costi R, Saunders A E, and Banin U 2010 Colloidal hybrid nanostructures: a new type of functional materials Angew. Chem. Int. Ed. 49, 4878

[10] Huynh W U, Dittmer J J, and Alivisatos A P 2002 Hybrid nanorod-polymer solar cells Science 29, 2425-2427

[11] Park B, Inoue T, Ogomi Y, Miyamoto A, Fujita1 S, Pandey S S, and Hayase S 2011 Electron Injection from Linearly Linked Two Dye Molecules to Metal Oxide Nanoparticles for Dye Sensitized Solar Cells Covering Wavelength Range from 400-950 nm Appl. Phys. Ex. 4, 012301

[12] Challe Kumar, Biofunctionalization of Nanomaterials (Wiley-VCH, 2005).

[13] Willner I, Basnar B and Willner B 2007 Nanoparticleenzyme hybrid systems for nanobiotechnology FEBS Journal 274, 302-309

[14] Quarta A, Di Corato R, Manna L, Ragusa A, and Pellegrino T 2007 Fluorescent-magnetic hybrid nanostructures: preparation, properties, and applications in biol- ogy IEEE Transactions on Nanobioscience 6, 4

[15] Rakovich A, Savateeva D, Rakovich T, Donegan J F, Rakovich Y P, Kelly V, Lesnyak V, Eychmüller A 2010 CdTe Quantum Dot/Dye Hybrid System as Photosensitizer for Photodynamic Therapy Nanoscale Res. Lett. 5, $753-760$

[16] Dulkeith E et al 2002 Fluorescence Quenching of Dye Molecules near Gold Nanoparticles: Radiative and Nonradiative Effects Phys. Rev. Lett. 89, 203002

[17] Lukomska J et al 2004 Fluorescence Enhancement of Fluorophores Tethered to Different Sized Silver Colloids Deposited on Glass Substrate Biopolymers 77, 31

[18] Cannone F et al 2006 Voltage Regulation of Fluorescence Emission of Single Dyes Bound to Gold Nanoparticles Nanoletters 7, 1070

[19] Gramotnev D K and Bozhevolnyi S I 2010 plasmonics beyond the diffraction limit NanoPhotonics 4, 83

[20] Hayat A, Nevet A, Ginzburg P, and Orenstein M 2011 Applications of two-photon processes in semiconductor photonic devices Semicond. Sci. Technol. 26, 083001

[21] Helmchen F and Denk W 2005 Deep tissue two-photon microscopy Nature Methods 2, 932

[22] Schuck P J, Fromm D P, Sundaramurthy A, Kino G S , and Moerner W E 2005 Improving the Mismatch between Light and Nanoscale Objects with Gold Bowtie Nanoantennas Phys. ReV. Lett. 94, 017402

[23] Bouhelier A, Bachelot R, Lerondel G, Kostcheev S, Royer P, and Wiederrecht G P 2005 Surface Plasmon Characteristics of Tunable Photoluminescence in Single Gold Nanorods Phys. Rev. Lett. 95, 267405

[24] Imura K, Nagahara T, and Okamoto H 2006 Photoluminescence from gold nanoplates induced by near-field two-photon absorption Appl. Phys. Lett. 88, 023104

[25] Lukomska J et al 2005 One- and Two-Photon Induced Fluorescence of Pacific Blue-Labeled Human Serum Albumin Deposited on Different Core Size Silver Colloids Biopolymers 81, 249

[26] Bates M, Huang B, Dempsey G T, Zhuang X 2007 Multicolor super-resolution imaging with photo-switchable fluorescent probes Science 317, 1749-1753

[27] Panchuk-Voloshina N, Haugland R P, Bishop-Stewart J, Bhalgat M K, Millard P J, Mao F, Leung W, and Haugland R P 1999 Alexa dyes, a series of new fluorescent dyes that yield exceptionally bright, photostable conjugates J. Histochem. Cytochem. 47(9), 1179-88

[28] Zhang Y, Yu J, Birch D J S, and Chen Y 2010 Gold nanorods for fluorescence lifetime imaging in biology $\mathrm{J}$. Bio. Opt. 15, 020504

[29] Zhang Y, Birch D J S, and Chen Y 2011 Two-photon ex- 
cited surface plasmon enhanced energy transfer between DAPI and gold nanoparticles: Opportunities in intracellular imaging and sensing Appl. Phys. Lett. 99, 103701

[30] Sen T, Sadhu S, and Patra A 2007 Surface energy transfer from rhodamine $6 \mathrm{G}$ to gold nanoparticles: A spectroscopic ruler Appl. Phys. Lett. 91, 043104

[31] Sen T, Jana S, Koner S, and Patra A 2010 Energy transfer between confined dye and surface attached $\mathrm{Au}$ nanoparticles of mesoporous silica J. Phys. Chem. C 114, 707

[32] Yun C S, Javier A, Jennings T, Fisher M, Hira S, Peterson S, Hopkins B, Reich N O, and Strouse G F 2005 Nanometal surface energy transfer in optical rulers, breaking the FRET barrier J. Am. Chem. Soc. 127, 3115
[33] Persson B N J and Lang N D 1982 Electron-hole-pair quenching of excited-states near a metal Phys. Rev. B 26(10), 5409

[34] Meystre P and Sargent M, Elements of Quantum Optics (Springer, Berlin, 2007).

[35] Sarid D and Challener W A, Modern Introduction to Surface Plasmons (Cambridge University Press, 2010).

[36] Nanotny L and Hecht B, Principles of Nano-optics (Cambridge University Press, 2006).

[37] Cox J D, Singh M R, Gumbs G, Anton M A, and Carreno F 2010 Dipole-dipole interaction between a quantum dot and a graphene nanodisk Phys. Rev. B 86, 123452 